\title{
16-Weeks of Combined Aerobic and Resistance Training and Hypo-Caloric Diet on Measures of Arterial Stiffness in Overweight Pre-Menopausal Women
}

Lisa M. Cotie, Andrea R. Josse, Stuart M. Phillips and Maureen J. MacDonald*

Department of Kinesiology, McMaster University, Hamilton ON.L8S 4K1, Canada

\begin{abstract}
Objective: While diet and exercise reduce the risk of cardiovascular morbidity in overweight populations, the effects of combined aerobic and resistance training on arterial health has not been comprehensively examined.

Methods: We investigated changes in both central and peripheral arterial stiffness using carotid artery distensibility and carotid to radial pulse wave velocity $\left(\mathrm{PWV}_{\mathrm{c-r}}\right)$, respectively, in twenty-five overweight, young women who participated in 16-weeks of 5-7 d/wk aerobic exercise, $2 \mathrm{~d} / \mathrm{wk}$ resistance training, and hypo-caloric diet intervention. Pro-Collagen Type I C-Peptide (PIP) was used as a marker of type I collagen synthesis and C-telopeptide of type I collagen (CTX), a marker of type I collagen degradation.

Results: Carotid artery distensibility was unaltered (Pre: $5.1 \times 10^{-3} \pm 3.9 \times 10^{-4}$ vs. Post: $5.5 \times 10^{-3} \pm 3.5 \times 10^{-4}, p=0.26$ ); however, $\mathrm{PWV}_{\mathrm{c-r}}$ increased following the intervention (Pre: $8.1 \pm 0.3 \mathrm{~m} / \mathrm{s}$ vs. Post: $8.9 \pm 0.3 \mathrm{~m} / \mathrm{s}, \mathrm{p}<0.05$ ). There were no changes in PIP (Pre: $1188 \pm 91 \mathrm{ng} / \mathrm{mL}$ vs. Post: $1222 \pm 94 \mathrm{ng} / \mathrm{mL}, \mathrm{p}=0.69$ ), however, CTX increased with the intervention $(0.65 \pm 0.01 \mathrm{ng} / \mathrm{mL}$ vs. Post: $0.80 \pm 0.02 \mathrm{ng} / \mathrm{mL}, \mathrm{p}<0.001)$. There were no relationships between markers of collagen turnover and arterial stiffness measures.

Conclusion: The intervention did not alter carotid artery distensibility or circulating markers of type I collagen synthesis but was associated with increased PWV and CTX. Therefore, 16-weeks of diet and combined aerobic and resistance training may lead to increased peripheral artery stiffness, as measured by $\mathrm{PWV}_{\mathrm{c}-\mathrm{r}}$, however, cardiovascular risk assessed by carotid artery distensibility remained unchanged.
\end{abstract}

Keywords: Combined aerobic and resistance training; Pulse wave velocity; Carotid artery distensibility; Obesity; Type I collagen

\section{Introduction}

Obesity has been identified as an independent risk factor for Cardiovascular Disease (CVD) [1,2]. Increased central artery stiffness, assessed by carotid artery compliance stiffness index and carotid-femoral Pulse Wave Velocity (PWV) is a strong predictor of cardiovascular disease risk in healthy and individuals at elevated risk $[3,4]$. Aerobic exercise has been shown to increase central arterial compliance and decrease carotid-femoral PWV and augmentation index and thus the risk of CVD in a variety of populations including young healthy men hypertensive men and women and both active and inactive pre- and postmenopausal women [5-9].

It is unclear whether these arterial health benefits are limited to exercise training programs that are primarily aerobic in nature or if other exercise modalities such as resistance training or combined aerobic and resistance training can decrease arterial stiffness. Rakobowchuk and colleagues found no change in carotid artery compliance after 6 and 12 weeks of whole body resistance training in young healthy men yet other studies have observed increases in both central and peripheral arterial stiffness after short duration resistance training in moderately active middle-aged men and in women with pre- and stage 1 hypertension $[8,10,11]$. There are minimal data, however, on the effects of combined aerobic and resistance training on arterial stiffness. Yang reported a decrease in whole body arterial stiffness (brachial-ankle PWV) in obese women (30-60 years) after three months of combined aerobic and resistance training [12]. The variations in methods and measurement sites in different studies likely contribute to the variability in outcomes with respect to the effects of different exercise training programs on arterial stiffness.

Information on circulating markers of collagen turnover have been used as proxies for arterial wall remodeling, for example using markers of type I collagen synthesis and degradation, may contribute to a better understanding of potential mechanisms responsible for changes in arterial stiffness observed with exercise/weight loss interventions [1316]. Few studies have investigated the relationship between arterial stiffness and markers of type I collagen synthesis and degradation and the results in the existing studies are mixed [13-16]. Some studies have reported positive associations between collagen synthesis and arterial stiffness or inverse relationships between collagen degradation and arterial stiffness while others have reported the opposite and found positive relationships between collagen degradation and arterial stiffness [13-15]. Clearly, more data are needed to comprehensively understand the role of type I collagen turnover in arterial stiffening and if markers of type 1 collagen turnover are useful markers of arterial stiffness.

Studies have shown that 10 weeks of aerobic exercise training does not change serum markers of type I collagen degradation (ICTP) or synthesis (PINP) in post-menopausal women [17]. Despite this there are no studies examining changes in arterial stiffness and collagen type 1 turnover with a combined exercise and diet intervention in pre-menopausal women who are at elevated risk for CVD. Thus, the purpose of this study was to determine the effects of a 16-week diet and combined aerobic and resistance exercise intervention on markers of type I collagen turnover and both central and peripheral measures of arterial stiffness, as assessed by carotid artery distensibility and

*Corresponding author: Maureen MacDonald, 1280 Main St. West, Hamilton, ON, Canada, L8S 4K1, Tel: 905 525-9140; Fax: 905 523-6011; E-mail macdonmj@mcmaster.ca

Received February 25, 2014; Accepted March 18, 2014; Published March 28, 2014

Citation: Cotie LM, Josse AR, Phillips SM, MacDonald MJ (2014) 16-Weeks of Combined Aerobic and Resistance Training and Hypo-Caloric Diet on Measures of Arterial Stiffness in Overweight Pre-Menopausal Women. J Metabolic Synd 3: 137. doi:10.4172/2167-0943.1000137

Copyright: $\odot 2014$ Cotie LM, et al. This is an open-access article distributed under the terms of the Creative Commons Attribution License, which permits unrestricted use, distribution, and reproduction in any medium, provided the original author and source are credited. 
carotid-radial PWV $\left(\mathrm{PWV}_{\mathrm{c-r}}\right)$ respectively, in obese and overweight premenopausal women. We hypothesized that both central and peripheral arterial stiffness would decrease and that there would be an associated increase in markers of collagen turnover following the 16-week weight loss intervention in young overweight women.

\section{Methods}

\section{Participants}

This study was part of a larger lifestyle intervention study: Improving Diet, Exercise and Lifestyle (I.D.E.A.L) for Women Study, which involved 90 participants [18]. A small subset of participants $(n=25)$ from the larger lifestyle intervention study volunteered to participate in the cardiovascular measurements for the current study, based on participant availability and consent. The Research Ethics Board of Hamilton Health Sciences approved the study. Twenty-five young, $(30 \pm 2$ years; mean \pm SE) obese (BMI: $32.4 \pm 0.7 \mathrm{~kg} / \mathrm{m}^{2}$ ) female subjects participated in this study. Other general inclusion criteria were: sedentary lifestyle, regular menstrual cycle, and no vitamin or mineral supplementation. Participants were deemed healthy and thus eligible to participate based on their responses to a short medical screening questionnaire and measurement of serum lipids, glucose, and insulin concentrations all of which were normal (data not shown). All participants provided written informed consent before participating in the study.

\section{Intervention}

As previously described, participants took part in both diet and exercise interventions over a 16 week period [18].

\section{Diet}

Participants were asked to consume a hypocaloric diet that was -750 $\mathrm{kcal} / \mathrm{d}$ less than their estimated requirements $(500 \mathrm{kcal} / \mathrm{d}$ by diet and $250 \mathrm{kcal} / \mathrm{d}$ through exercise). Maintenance energy requirements were calculated for each participant using the Mifflin St Jeor equation with a sedentary activity factor [19]. All participants received individualized diet counseling by study dieticians and research nutritionists on a biweekly basis. Every 2 weeks, participants provided a 3-d food record to track compliance. Participants were divided into three dietary groups, based on dairy intake, throughout the 16-weeks. The dietary specifications of the three groups have been previously described [18]. Briefly, the groups included a) Adequate Protein, Low Dairy (APLD) b) Adequate Protein, Medium Dairy (APMD) and c) High Protein, High Dairy (HPHD). Of the twenty-five participants in this study, eight were in APLD, eight in APMD and nine in HPHD.

\section{Exercise training}

Participants completed 16 weeks of combined aerobic and resistance training as part of a targeted body composition-changing protocol. Participants exercised at the main fitness centre at McMaster University. They engaged in a number of modes of aerobic exercise (stationary cycling, jogging on a treadmill, walking on an indoor track) $5 \mathrm{~d} / \mathrm{wk}$ and resistance exercise $2 \mathrm{~d} / \mathrm{wk}$ with supervision. Each exercise session was designed to result in the expenditure of $250 \mathrm{kcal}$. During the week (Monday - Friday), participants reported to the study office and were given a SenseWear Pro (BodyMedia, Pittsburgh, PA, US) energy expenditure arm band device to track energy expenditure [20]. Participants were requested to wear the SenseWear Pro device at home on random occasions in order to assess compliance with weekend workouts. In addition to aerobic exercise, participants also completed a progressive resistance training program $2 \mathrm{~d} / \mathrm{wk}$ (upper body, lower body split) with trained study personnel. The weight lifted by each participant was recorded after each session and was increased when the participant was able to complete 3 sets of 10 repetitions or more at any given weight. The trainers completed exercise logs after the resistance exercise sessions and the participant after the aerobic sessions. These were checked frequently to ensure compliance to the program. The aerobic and resistance training programs have been described previously [18].

\section{Cardiovascular Measurements}

\section{Heart rate and blood pressure}

Assessment sessions were conducted before and after the intervention and began with 10 minutes of supine rest to ensure representative resting measurements prior to the commencement of the vascular assessment. Continuous measurements of heart rate via single lead Electrocardiograph (ECG) (model ML123, AD Instruments Inc. Colorado Springs, Colo.) and brachial Blood Pressure (BP) measurements via an automated applanation tonometer with oscillometric cuff calibration (model CBM-7000; Colin Medical Instruments, San Antonio, TX.) were made. All analogue signals were converted to digital using a fast Fourier transform and were sampled simultaneously at $200 \mathrm{~Hz}$ using a commercially available data acquisition system (Power lab Model ML 795, ADInstruments, Colorado Springs, USA) and software program (LabChart 7.0; ADInstruments Inc. Colorado Springs, CO, USA).

\section{Arterial Stiffness Measures}

\section{Peripheral artery stiffness}

Peripheral artery stiffness was estimated using $\mathrm{PWV}_{\mathrm{c}-\mathrm{r}}$ using high fidelity, simultaneous carotid and radial artery pressure measurements. $\mathrm{PWV}_{\mathrm{c}-\mathrm{r}}$ was defined as the time delay in arrival of the foot of the pulse wave between the carotid and radial arteries. Arterial waveforms at the common carotid and radial arteries were collected using a handheld tonometer (model SPT-301, Millar Instruments Inc., Houston, TX) positioned on the right common carotid and radial artery at the point of greatest pulsation to produce continuous arterial pressure waveforms. Peripheral pulse transit time was determined using the subtraction method [21]. This was achieved by determining the time delay between ventricular depolarization (R-wave peak), and the "foot" of the radial and carotid artery waveforms. The pressure waveforms obtain were band-pass filtered $(5-30 \mathrm{~Hz})$ with the lower $(\leq 5 \mathrm{~Hz})$ and higher frequencies $(\geq 30 \mathrm{~Hz})$ removed in order to assist in the detection of the foot of each waveform. The foot of each waveform was identified as the minimum value of the digitally filtered signal and corresponded to the end of diastole, when the steep rise in the wave begins and appears as a sharp inflection of the original signal [22].

Similarly the path length between the pulse measurement sites was calculated by subtracting the surface distance between the sternal notch and the carotid tonometer placement from that of the sternal notch and the radial tonometer placement. An anthropometric measuring tape was used to measure the straight-line distance between skin sites along the surface of the body. Resting PWV data was recorded during 20 continuous heart cycles using the equation:

Equation 1: $P W V_{c-r}=\frac{D t}{\Delta T}$

Where $\mathrm{D}$ is the distance between measurement sites, and $\mathrm{t}$ is the pulse transit time.

\section{Central artery stiffness}

Central artery stiffness was estimated with direct measurements of carotid artery distensibility, as previously described using a combination 
of high-resolution, two-dimensional, brightness mode ultrasound images (SystemFiVe; GE Medical Systems, Horten, Norway) and applanation tonometry (model SPT-301; Millar Instruments, Houston, TX, USA) [23]. Common carotid artery images were collected using a $10 \mathrm{MHz}$ ultrasound probe and were collected at $10 \mathrm{frames} \mathrm{sec}^{-1}$. A handheld tonometer was positioned over the point of greatest pulsation of the right common carotid artery and held in a fixed position for ten consecutive heart cycles while ultrasound images of the left common carotid artery were collected simultaneously. Absolute carotid artery systolic blood pressures were calculated by calibrating the relative values acquired using applanation tonometry to the calibrated brachial artery blood pressure acquired simultaneously [24,25]. Ultrasound images were stored offline in Digital Image and Communications in Medicine (DICOM) format for later analysis using semi-automated edge tracking system [AMS, (Artery Measurement System) Image and Data Analysis: Tomas Gustavsson]. In each frame, carotid (minimum, mean and maximum) lumen diameters were calculated from roughly 100 measurement markers along the vessel wall within a chosen region of interest, for a total of 110,000 measures in the 10 heart cycles. Distensibility was calculated using the following equations [26].

$$
\text { Equation 2: } \text { Distensibility }=\frac{\left[\Pi\left(\frac{d_{\max }}{2}\right)^{2} \Pi\left(\frac{d_{\min }}{2}\right)^{2}\right]}{\Pi\left(\frac{d_{\min }}{2}\right)^{2} \times P P}
$$

Where $d_{\max }$ is the maximum diameter, $d_{\text {min }}$ is the minimum diameter, and PP is carotid pulse pressure, the change in pressure DBP and SBP. The mean carotid diameter was calculated using the average of all diameters acquired throughout the ten heart cycles.

\section{Blood analysis}

Overnight fasted venous blood samples were collected prior to and after 16 weeks of the intervention for further analysis. Serum was stored at $-20^{\circ} \mathrm{C}$ for further analysis. Serum samples were analyzed for levels of pro-collagen type I (PIP) as a marker of type 1 collagen synthesis (Takara, Mountain View, CA) and cross-linked telopeptide of type I collagen (CTX) as a marker of degradation (Immunodiagnostic Systems, Scottsdale, AZ) using the corresponding ELISA kits.

\section{Strength and aerobic fitness testing}

As described previously strength was tested by determining the participants' single lift voluntary maximal strength using leg extension, hamstring curl, seated row and chest press before and after the intervention [18]. These tests were completed under the supervision of study personnel. Aerobic fitness was measured using a modified Astrand submaximal fitness protocol. The participants were asked to cycle on a stationary bicycle (Monark Ergomedic 828E, Health Care International) at two consecutive workloads for 6 minutes each and HR was recorded every minute.

\section{Statistics}

Results are presented as mean \pm SE and differences were considered significant at $\mathrm{p}<0.05$. Data were analyzed using SPSS (Version 11.5 for Windows). The three dietary groups were compared for difference in all measures. No differences were observed and thus the data was pooled for all further analysis. The effect of the intervention (pre vs. post) was examined using paired t-tests. Possible relationships between the blood markers and functional markers of arterial stiffness were analyzed using Pearson correlation coefficient.

\section{Results}

There were no differences in any of the vascular or blood marker measures between the dietary groups; therefore all dietary groups were pooled for subsequent analysis.

\section{Participants}

A total of twenty-five $(\mathrm{n}=25)$ young healthy women (mean $[ \pm \mathrm{SE}]$ age (years) $=30 \pm 2$; height $(\mathrm{cm})=163 \pm 1)$ completed the vascular assessments before and after the lifestyle intervention. Following the 16-week intervention body mass and BMI decreased significantly (Table 1).

\section{Intervention Data}

Strength was increased significantly following the intervention as measured by seated row, chest press; leg extension and hamstring curl (Table 1). Aerobic fitness improved significantly following the intervention as measured by a mean HR reduction of $15 \mathrm{bpm}$ during the modified Astrand protocol from pre to post intervention $(\mathrm{p}<0.001)$.

\section{Cardiovascular Data}

There were no changes in supine resting heart rate, MAP, SBP or DBP following the intervention (Table 1).

\section{Arterial Stiffness}

$\mathrm{PWV}_{c-\mathrm{r}}$ (Figure 1A) was increased after the 16-week diet and exercise intervention (Pre: $8.1 \pm 0.3 \mathrm{~m} / \mathrm{s}$ vs. Post: $8.9 \pm 0.3 \mathrm{~m} / \mathrm{s}, \mathrm{p}<0.05$ ).

\begin{tabular}{|c|c|c|c|}
\hline Variable & Pre & Post & P value \\
\hline Body Mass $(\mathrm{kg})$ & $86.8 \pm 2.4$ & $80.6 \pm 2.4$ & $<0.001$ \\
\hline BMI $\left(\mathrm{kg} / \mathrm{m}^{2}\right)$ & $32.4 \pm 0.8$ & $30.1 \pm 0.7$ & $<0.001$ \\
\hline Resting Supine HR (bpm) & $65 \pm 1$ & $62 \pm 1$ & $>0.05$ \\
\hline Resting Supine MAP (mmHg) & $81 \pm 2$ & $81 \pm 3$ & $>0.05$ \\
\hline Resting Supine SBP (mmHg) & $116 \pm 2$ & $114 \pm 3$ & $>0.05$ \\
\hline Resting Supine DBP (mmHg) & $63 \pm 2$ & $63 \pm 3$ & $>0.05$ \\
\hline Seated Row (lbs) & $79.6 \pm 2.9$ & $89.2 \pm 2.8$ & $<0.001$ \\
\hline Chest Press (lbs) & $63.3 \pm 3.5$ & $71.7 \pm 2.7$ & 0.001 \\
\hline Leg Extension (lbs) & $180.0 \pm 7.5$ & $203.6 \pm 6.2$ & $<0.001$ \\
\hline Hamstring Curl (lbs) & $108.0 \pm 4.4$ & $122.4 \pm 4.2$ & $<0.001$ \\
\hline $\begin{array}{c}\text { Mean Heart Rate (bpm) during } \\
\text { modified Åstrand Test }\end{array}$ & $148 \pm 3$ & $133 \pm 3$ & $<0.001$ \\
\hline
\end{tabular}

Table 1: Participant Characteristics.
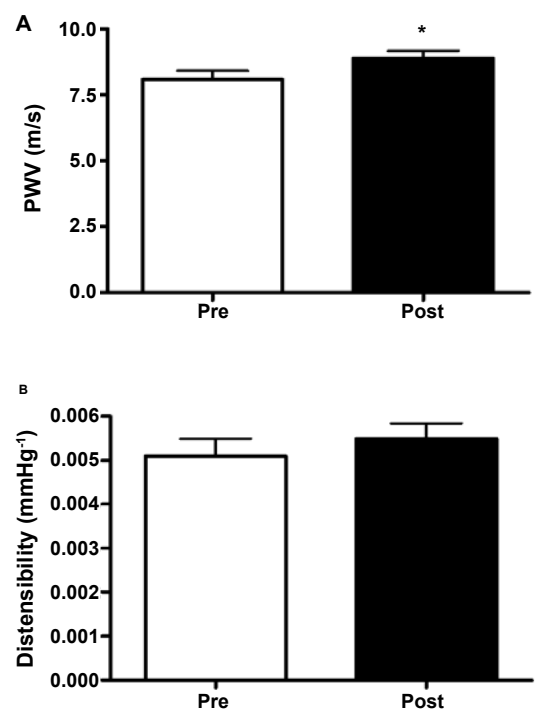

Figure 1: Changes in $\mathrm{PWV}_{\mathrm{cr}}$ and carotid artery distensibility from Week 0 to Week 16. 
Conversely, carotid artery distensibility (Figure 1B) was unchanged with the intervention (Pre: $5.1 \times 10^{-3} \pm 3.9 \times 10^{-4}$ vs. Post: $5.5 \times 10^{-3} \pm$ $\left.3.5 \times 10^{-4}, \mathrm{p}=0.26\right)$.

\section{Relationship between peripheral and central artery stiffness}

There was no relationship observed between measures of $\mathrm{PWV}_{\mathrm{c}-\mathrm{r}}$ and carotid artery distensibility (Figure 2).

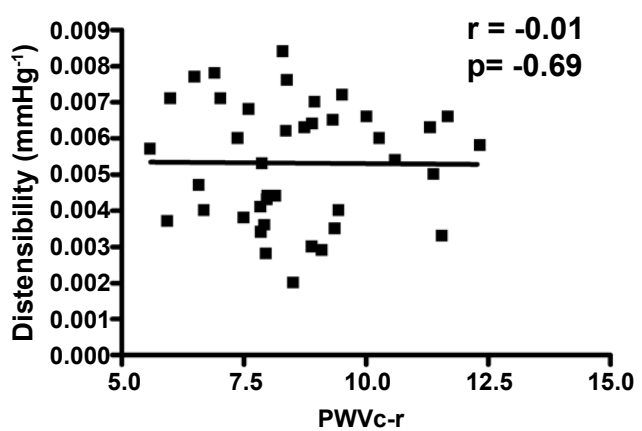

Figure 2: Relationship between peripheral and central artery stiffness.

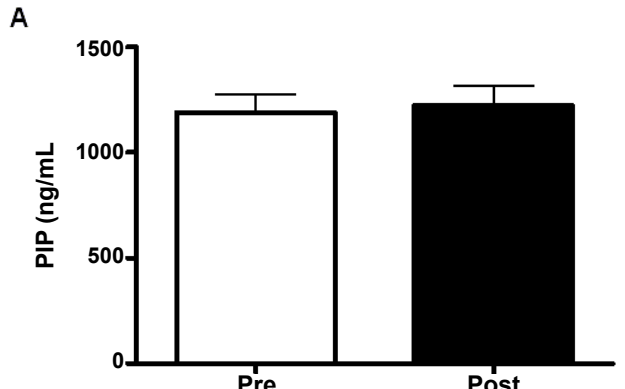

B

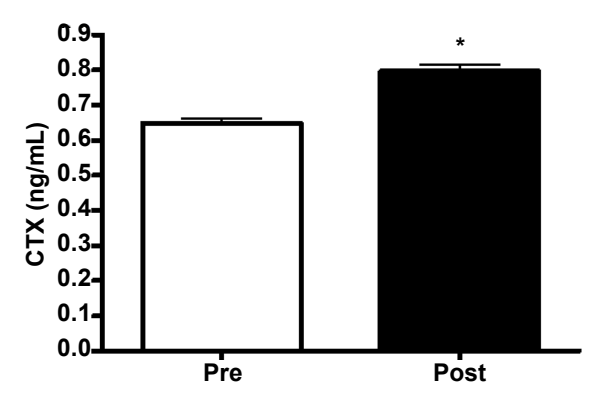

C

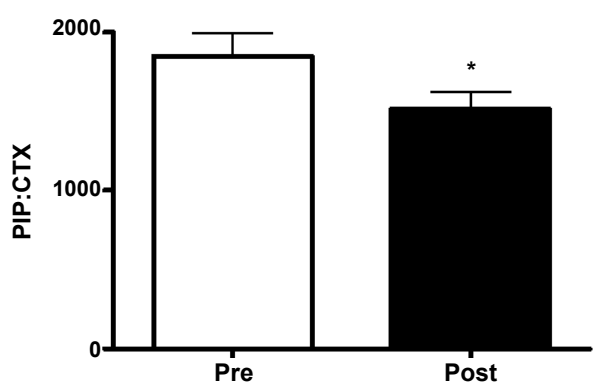

Figure 3: Changes in serum type I collagen markers from Week 0 to Week 16.

\section{Type I collagen markers}

As a marker of collagen synthesis PIP was unchanged (Pre: 1188 $\pm 91 \mathrm{ng} / \mathrm{mL}$ vs. Post: $1222 \pm 94 \mathrm{ng} / \mathrm{mL}, \mathrm{p}=0.69$; Figure $3 \mathrm{~A}$ ) after the 16-week intervention. However, there was a main effect of time for a marker of collagen degradation, CTX, (Pre: $0.65 \pm 0.01 \mathrm{ng} / \mathrm{mL}$ vs. Post: $0.80 \pm 0.02 \mathrm{ng} / \mathrm{mL}, \mathrm{p}<0.001$; Figure $3 \mathrm{~B})$. The ratio of collagen markers (PIP: CTX), used previously to represent collagen turnover, decreased with the intervention (Pre: $1851.6 \pm 140.8$ vs. Post: $1514.7 \pm 111.6, p=$ 0.008 ; Figure 3C). All serum ELISA kits had coefficients of variance of $<10 \%$.

\section{Relationship between type I collagen markers and arterial stiffness}

There were no relationships observed between either the central or peripheral artery stiffness measures or the type I collagen markers or the ratio of those markers.

\section{Discussion}

Our research shows that in overweight and obese pre-menopausal women a 16 week lifestyle intervention combining aerobic and resistance exercise training and a hypocaloric diet did not change central artery stiffness, or resting blood pressure, but decreased BMI and increased upper limb arterial stiffness. While literature suggests that weight loss or exercise training interventions can result in decreased arterial stiffness, there is little information about the impact of combined diet and exercise lifestyle interventions on this independent predictor of cardiovascular mortality.

We believe the regional variations we observed in arterial stiffness changes may help to explain the existing variability in literature with respect to the impact of different exercise/weight loss interventions on arterial stiffness. Central artery stiffness, measured via carotid to femoral PWV or direct carotid distensibility, is an accepted predictor for CVD risk, particularly in populations at risk for CVD [27]. Peripheral artery stiffness may provide valuable information about the mechanisms regulating vascular remodeling and may be an acceptable surrogate for central stiffness in some populations [27]. However, when comparing and contrasting different research studies in this area it may not be appropriate to equate measures of whole body stiffness (brachial- ankle PWV), central arterial stiffness (carotid distensibility or carotid-femoral PWV), peripheral arterial stiffness (carotid-radial PWV or femoral-dorsalis pedis PWV) and calculated arterial stiffness indices (augmentation index).

In the current study we were interested in determining if $\mathrm{PWV}_{\mathrm{c}-\mathrm{r}}$ would be a good surrogate for central artery stiffness, Measurements of carotid to femoral PWV were a concern since this gold standard measure of central PWV involves exposure of the inguinal region for the femoral site measurement. In our population of overweight and obese women, measurements of arterial pressure at the femoral site generally are difficult to conduct due to their distribution of body fat and the associated discomfort of the participants during assessment. Our results demonstrate that despite the positive weight loss outcomes, this multi-faceted exercise and diet-based intervention led to diverse arterial structural changes that were dependent on the arterial segment examined and the technique used. These findings further suggest that peripheral PWV measures are likely not an acceptable surrogate for central stiffness measures in this population.

Regardless of assessment method, it is consistently reported that weight loss or aerobic exercise training alone or in combination result in decreased indices of central arterial stiffness $[9,28,29]$. Of concern 
are the potential detrimental cardiovascular effects of resistance exercise training as there have been reports of increased decreased and no change in arterial stiffness with resistance exercise training in women [28,30-34]. Some of the apparently disparate results may be due to the different assessment methods, different intensities of resistance training employed and variations in baseline arterial stiffness of the participants. Despite these concerns current guidelines for individuals with cardiovascular disease include resistance exercise components and a recent meta-analysis highlighted the positive impact of resistance exercise training on cardiovascular risk factors $[35,36]$.

Current exercise recommendations commonly incorporate combined aerobic and resistance exercise training methods, yet little previous research has examined the vascular impacts of combined training interventions. Yang reported a decrease in whole body (brachial-ankle PWV) in overweight women (30-60 years) after three months of combined aerobic and resistance training however it is difficult to translate these findings to assessments of CV risk as it is not clear if whole body PWV is indicative of central PWV [12]. Figueroa et al. observed a decrease in brachial-ankle PWV following a 12-week moderate-intensity combined circuit of resistance and endurance exercise training in post menopausal women [37]. Another study by the same group in 2013 demonstrated that a hypo-caloric diet alone or in combination with light resistance exercise training resulted in similar changes in brachial-ankle PWV while Ho et al. observed a decrease in augmentation index after 12 weeks of combined aerobic and resistance training in overweight an obese adults $[28,38]$. Interestingly, in the current study, peripheral PWV was increased with the intervention but like whole body PWV, it is also not clear if that is an acceptable indication of changes in cardiovascular risk as risk prediction is currently only linked to central artery stiffness which in our study remained unchanged [27].

Previous research examining the impact of weight loss achieved exclusively through caloric restriction interventions supports the concept that weight loss is associated with decreased central arterial stiffness in young healthy adults and in overweight and obese middle aged and older men and women $[39,40]$. The study by Dengo et al. assessed both central PWV and carotid stiffness and found both indices of central arterial stiffness to decrease in the weight loss but not the control group. In the current study, we found no change in carotid artery stiffness with the combined diet and exercise intervention, while our weight loss magnitudes were comparable to those of Dengo ( $\sim 7 \mathrm{Kg}$ over 12 weeks). Balkestein at al. found in a group of healthy obese men that carotid artery stiffness was decreased with weight loss with or without exercise and that exercise did not have an additive effect [41]. Due to design limitations, these previous studies were unable to examine sex or age differences and the exclusive female cohort and unique nature of the lifestyle intervention in the current study may account for some of the differences observed in comparison to the literature.

Changes in elastin or collagen material content or arrangement mechanisms have been suggested to contribute to the reductions in arterial stiffness with lifestyle interventions. In an effort to determine mechanisms associated with changes in arterial stiffness, we measured the effects of our intervention on blood markers of type I collagen synthesis and degradation, as others have done and looked at the relationships to the measures of both central and peripheral artery stiffness [13-16]. Pro-collagen type I, an indicator of type I collagen synthesis was unchanged after the intervention and this finding is consistent with other reports [17]. To our knowledge measures of type I collagen synthesis following a long-term (16-week) diet and combined aerobic and resistance - exercise program have not been made in pre- menopausal women. Studies have, however, shown that long-term low and high intensity aerobic training do not result in changes in markers of type I collagen degradation (CTX or ICTP) in post-menopausal women $[17,42]$. In contrast, we observed an increase in type I collagen degradation (CTX) following the 16-week intervention. The ratio of PIP: CTX, thought to be representative of type I collagen turnover, was also unchanged after the16-week interventions.

Conflicting relationships between arterial stiffness and type I collagen degradation have been reported in the literature [13-15]. We did not find a relationship between either type I collagen synthesis or degradation and our measures of either central or peripheral artery stiffness. It is possible our sample size of 25 was not large enough to see this relationship, as other studies have had larger sample sizes $(\mathrm{N}=46-80)$, however, we did not even observe noticeable trends in our data [13-15]. It is also possible that the degree of vascular remodeling, if it occurred, was relatively small in our young obese, but otherwise healthy, women who may not have had extensive arterial dysfunction to begin with. Circulating markers of collagen synthesis/degradation likely reflect turnover of pools of collagen distinct from that present only in arteries (i.e., bone and muscle) and thus cannot, particularly in an exercise intervention, be used as a reflection of solely arterial remodeling.

Our study demonstrates that despite weight loss following a 16week diet and combined aerobic and resistance training intervention peripheral stiffness may be increased. It is important to consider the duration and mode of exercise and diet employed and the overall balance of cardiovascular risk outcomes when assessing the total benefits of lifestyle interventions. This study does not support the use of type 1 collagen markers as an index over either central or peripheral artery stiffness. Further research is warranted to better understand these relationships and the mechanisms responsible for the observed changes.

\section{Acknowledgements}

Funding for this study was supplied by Natural Science and Engineering Research Council of Canada Discovery grants (MacDonald and Phillips), Canadian Institutes of Health Research (CGS to Josse and Operating grant to Phillips), The Dairy Research Institute and the Dairy Farmers of Canada.

\section{References}

1. Zebekakis PE, Nawrot T, Thijs L, Balkestein EJ, van der Heijden-Spek J, et al. (2005) Obesity is associated with increased arterial stiffness from adolescence until old age. J Hypertens 23: 1839-1846.

2. Grundy SM (1998) Multifactorial causation of obesity: implications for prevention. Am J Clin Nutr 67: 563S-72S.

3. Danias PG, Tritos NA, Stuber M, Botnar RM, Kissinger KV, et al. (2003) Comparison of aortic elasticity determined by cardiovascular magnetic resonance imaging in obese versus lean adults. Am J Cardiol 91: 195-199.

4. Wildman RP, Mackey RH, Bostom A, Thompson T, Sutton-Tyrrell K (2003) Measures of obesity are associated with vascular stiffness in young and older adults. Hypertension 42: 468-473.

5. Tanaka H, Dinenno FA, Monahan KD, Clevenger CM, DeSouza CA, et al (2000) Aging, habitual exercise, and dynamic arterial compliance. Circulation 102: $1270-1275$

6. Vaitkevicius PV, Fleg JL, Engel JH, O'Connor FC, Wright JG, et al. (1993) Effects of age and aerobic capacity on arterial stiffness in healthy adults. Circulation 88: 1456-1462.

7. Kakiyama T, Sugawara J, Murakami H, Maeda S, Kuno S, et al. (2005) Effects of short-term endurance training on aortic distensibility in young males. Med Sci Sports Exerc 37: 267-271.

8. Collier SR, Kanaley JA, Carhart R Jr, Frechette V, Tobin MM, et al. (2008) Effect of 4 weeks of aerobic or resistance exercise training on arterial stiffness, blood flow and blood pressure in pre- and stage-1 hypertensives. J Hum Hypertens 22: $678-686$ 
Citation: Cotie LM, Josse AR, Phillips SM, MacDonald MJ (2014) 16-Weeks of Combined Aerobic and Resistance Training and Hypo-Caloric Diet on Measures of Arterial Stiffness in Overweight Pre-Menopausal Women. J Metabolic Synd 3: 137. doi:10.4172/2167-0943.1000137

Page 6 of 6

9. Tanaka H, DeSouza CA, Seals DR (1998) Absence of age-related increase in central arterial stiffness in physically active women. Arterioscler Thromb Vasc Biol 18: 127-132.

10. Rakobowchuk M, McGowan CL, de Groot PC, Bruinsma D, Hartman JW, et al (2005) Effect of whole body resistance training on arterial compliance in young men. Exp Physiol 90: 645-651.

11. Collier SR, Frechette V, Sandberg K, Schafer P, Ji H, et al. (2011) Sex differences in resting hemodynamics and arterial stiffness following 4 weeks of resistance versus aerobic exercise training in individuals with pre-hypertension to stage 1 hypertension. Biol Sex Differ 2: 9

12. Yang SJ, Hong HC, Choi HY, Yoo HJ, Cho GJ, et al. (2011) Effects of a threemonth combined exercise programme on fibroblast growth factor 21 and fetuin-A levels and arterial stiffness in obese women. Clin Endocrinol (Oxf) 75 464-469.

13. Chatzikyriakou SV, Tziakas DN, Chalikias GK, Stakos DA, Thomaidi AK, et al. (2008) Serum levels of collagen type-I degradation markers are associated with vascular stiffness in chronic heart failure patients. Eur J Heart Fail 10: 11811185

14. Ishikawa J, Kario K, Matsui Y, Shibasaki S, Morinari M, et al. (2005) Collagen metabolism in extracellular matrix may be involved in arterial stiffness in older hypertensive patients with left ventricular hypertrophy. Hypertens Res 28: 9951001.

15. McNulty M, Mahmud A, Spiers P, Feely J (2006) Collagen type-I degradation is related to arterial stiffness in hypertensive and normotensive subjects. J Hum Hypertens 20: 867-873.

16. Stakos DA, Tziakas DN, Chalikias GK, Mitrousi K, Tsigalou C, et al. (2010) Associations between collagen synthesis and degradation and aortic function in arterial hypertension. Am J Hypertens 23: 488-494.

17. Cornelissen VA, Fagard RH, Lijnen PJ (2010) Serum collagen-derived peptides are unaffected by physical training in older sedentary subjects. J Sci Med Sport 13: $424-428$

18. Josse AR, Atkinson SA, Tarnopolsky MA, Phillips SM (2011) Increased consumption of dairy foods and protein during diet- and exercise-induced weight loss promotes fat mass loss and lean mass gain in overweight and obese premenopausal women. J Nutr 141: 1626-1634.

19. Frankenfield D, Roth-Yousey L, Compher C (2005) Comparison of predictive equations for resting metabolic rate in healthy nonobese and obese adults: a systematic review. J Am Diet Assoc 105: 775-789.

20. Welk GJ, McClain JJ, Eisenmann JC, Wickel EE (2007) Field validation of the MTI Actigraph and BodyMedia armband monitor using the IDEEA monitor. Obesity (Silver Spring) 15: 918-928

21. Weber T, Ammer M, Rammer M, Adji A, O'Rourke MF, et al. (2009) Noninvasive determination of carotid-femoral pulse wave velocity depends critically on assessment of travel distance: a comparison with invasive measurement. $J$ Hypertens 27: 1624-1630.

22. Munakata M, Ito N, Nunokawa T, Yoshinaga K (2003) Utility of automated brachial ankle pulse wave velocity measurements in hypertensive patients. Am J Hypertens 16: 653-657.

23. Currie KD, Proudfoot NA, Timmons BW, MacDonald MJ (2010) Noninvasive measures of vascular health are reliable in preschool-aged children. Appl Physiol Nutr Metab 35: 512-517.

24. Nichols WW, Hartley C, McDonald DA, O'Rourke MF (1998) McDonald's blood flow in arteries: theoretic, experimental, and clinical principles, (4thedn,) Arnold; Oxford University Press, London, New York, pp: 564.

25. Kelly R, Fitchett D (1992) Noninvasive determination of aortic input impedance and external left ventricular power output: a validation and repeatability study of a new technique. J Am Coll Cardiol 20: 952-963.
26. O'Rourke MF, Staessen JA, Vlachopoulos C, Duprez D, Plante GE (2002) Clinical applications of arterial stiffness; definitions and reference values. Am J Hypertens 15: 426-444

27. Laurent S, Cockcroft J, Van Bortel L, Boutouyrie P, Giannattasio C, et al. (2006) Expert consensus document on arterial stiffness: methodological issues and clinical applications. Eur Heart J 27: 2588-2605.

28. Ho SS, Radavelli-Bagatini S, Dhaliwal SS, Hills AP, Pal S (2012) Resistance, aerobic, and combination training on vascular function in overweight and obese adults. J Clin Hypertens (Greenwich) 14: 848-854.

29. Satoh N, Shimatsu A, Kato Y, Araki R, Koyama K, et al. (2008) Evaluation of the cardio-ankle vascular index, a new indicator of arterial stiffness independent of blood pressure, in obesity and metabolic syndrome. Hypertens Res 31: 19211930.

30. Rossow LM, Fahs CA2, Thiebaud RS3, Loenneke JP4, Kim D5, et al. (2014) Arterial stiffness and blood flow adaptations following eight weeks of resistance exercise training in young and older women. Exp Gerontol

31. Cortez-Cooper MY, DeVan AE, Anton MM, Farrar RP, Beckwith KA, et al (2005) Effects of high intensity resistance training on arterial stiffness and wave reflection in women. Am J Hypertens 18: 930-934.

32. Okamoto T, Masuhara M, Ikuta K (2006) Effects of eccentric and concentric resistance training on arterial stiffness. J Hum Hypertens 20: 348-354

33. Williams AD, Ahuja KD, Almond JB, Robertson IK, Ball MJ (2013) Progressive resistance training might improve vascular function in older women but not in older men. J Sci Med Sport 16: 76-81.

34. Yoshizawa M, Maeda S, Miyaki A, Misono M, Saito Y, et al. (2009) Effect of 12 weeks of moderate-intensity resistance training on arterial stiffness: a randomised controlled trial in women aged 32-59 years. Br J Sports Med 43 615-618.

35. Williams, MA, Haskell WL, Ades PA, Amsterdam EA, Bittner V, et al. (2007) Resistance exercise in individuals with and without cardiovascular disease 2007 update: A scientific statement from the American Heart Association Council on Clinical Cardiology and Council on Nutrition, Physical Activity, and Metabolism. Circulation, 116: 572-584

36. Cornelissen VA, Fagard RH, Coeckelberghs E, Vanhees L (2011) Impact of resistance training on blood pressure and other cardiovascular risk factors: a meta-analysis of randomized, controlled trials. Hypertension 58: 950-958.

37. Figueroa A, Park SY, Seo DY, Sanchez-Gonzalez MA, Baek YH (2011) Combined resistance and endurance exercise training improves arteria stiffness, blood pressure, and muscle strength in postmenopausal women. Menopause 18: 980-984

38. Figueroa A, Wong A, Kinsey A, Kalfon R, Eddy W, et al. (2014) Effects of milk proteins and combined exercise training on aortic hemodynamics and arteria stiffness in young obese women with high blood pressure. Am J Hypertens 27 : 338-344.

39. Wildman RP, Farhat GN, Patel AS, Mackey RH, Brockwell S, et al. (2005) Weight change is associated with change in arterial stiffness among healthy young adults. Hypertension 45: 187-192.

40. Dengo AL, Dennis EA, Orr JS, Marinik EL, Ehrlich E, et al. (2010) Arteria destiffening with weight loss in overweight and obese middle-aged and older adults. Hypertension 55: 855-861.

41. Balkestein EJ, van Aggel-Leijssen DP, van Baak MA, Struijker-Boudier HA, Van Bortel LM (1999) The effect of weight loss with or without exercise training on large artery compliance in healthy obese men. J Hypertens 17: 1831-1835.

42. Bergström I, Parini P, Gustafsson SA, Andersson G, Brinck J (2012) Physical training increases osteoprotegerin in postmenopausal women. J Bone Mine Metab 30: 202-207.
This article was originally published in a special issue, Cardiovascular Risks Associated with Metabolic syndrome handled by Editor(s). Dr. Gen-Min Lin Department of Medicine, Hualien-Armed Forces General Hospital, China 